Biomarkers

\title{
Comparison of $\beta 2$-microglobulin serum level between Alzheimer's patients, cognitive healthy and mild cognitive impaired individuals
}

Roberto Dominici, Dario Finazzi, Letizia Polito, Emanuela Oldoni, Giovanna Bugari, Alessandro Montanelli, Elio Scarpini, Daniela Galimberti \& Antonio Guaita

To cite this article: Roberto Dominici, Dario Finazzi, Letizia Polito, Emanuela Oldoni, Giovanna Bugari, Alessandro Montanelli, Elio Scarpini, Daniela Galimberti \& Antonio Guaita (2018) Comparison of $\beta 2$-microglobulin serum level between Alzheimer's patients, cognitive healthy and mild cognitive impaired individuals, Biomarkers, 23:6, 603-608, DOI: 10.1080/1354750X.2018.1468825

To link to this article: https://doi.org/10.1080/1354750X.2018.1468825

Published online: 23 May 2018.

Џll Article views: 225

View Crossmark data ¿
Submit your article to this journal $₫$

Q View related articles $ک$

4 Citing articles: 4 View citing articles $₫$ 


\title{
Comparison of $\beta 2$-microglobulin serum level between Alzheimer's patients, cognitive healthy and mild cognitive impaired individuals
}

\author{
Roberto Dominici ${ }^{\mathrm{a}}$, Dario Finazzi ${ }^{\mathrm{b}, c *} \mathbb{D}$, Letizia Polito ${ }^{\mathrm{d}}$, Emanuela Oldoni ${ }^{\mathrm{e}}$ (D) , Giovanna Bugari $^{\mathrm{b}}$, \\ Alessandro Montanelli ${ }^{b}$, Elio Scarpini ${ }^{\mathrm{e}}$ (D), Daniela Galimberti ${ }^{\mathrm{e}}$ (D) and Antonio Guaita ${ }^{\mathrm{d}}$ \# (iD)

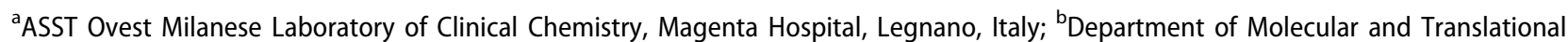 \\ Medicine, University of Brescia, Brescia, Italy; 'Clinical Chemistry Laboratory, Diagnostic Department, ASST Spedali Civili di Brescia, Brescia, \\ Italy; ${ }^{d}$ Fondazione Golgi-Cenci Abbiategrasso (MI), Milano, Italy; ${ }^{e}$ Neurodegenerative Disease Unit, Department of Pathophysiology and \\ Transplantation, "Dino Ferrari" Center, University of Milan, Fondazione Cà Granda, IRCCS Ospedale Maggiore Policlinico, Milan, Italy
}

\begin{abstract}
Background: Several studies performed in the last years on the brain, showed that beta2-microglobulin $(\beta 2 \mathrm{~m})$ and $\mathrm{MHC}$ can act independently of their canonical immune function to regulate normal brain development, synaptic plasticity and behaviour. Increased systemic levels of soluble $\beta 2 \mathrm{~m}$ have been implicated in cognitive impairments like that associated with chronic haemodialysis, or aortic valve replacement. Increased soluble $\beta 2 \mathrm{~m}$ has also been detected in the cerebral spinal fluid (CSF) of patients with HIV-associated dementia and Alzheimer's disease (AD).

Objective: To compare plasma $\beta 2 \mathrm{~m}$ levels in healthy subjects and subjects with dementia or cognitive impairment.

Methods: We measured the concentration of $\beta 2 \mathrm{~m}$ in a cohort of 245 individuals and compared sex matched, cognitive healthy individuals.

Results: We found higher levels of $\beta 2 \mathrm{~m}$ in $A D$ patients compared to non- $A D \mathrm{MCl}$ and healthy controls ( $2063 \mathrm{ng} / \mathrm{mL} \pm 852$ versus $1613 \pm 503$ and $1832 \pm 382 \mathrm{ng} / \mathrm{mL}, p<0.001$ and $<0.033$, respectively), while there was no difference between mild cognitive impairment $(\mathrm{MCl})$ and healthy controls $(p>0.05)$.

Conclusions: Our data confirm that $\beta 2 \mathrm{~m}$ could play a role in $A D$. However, a replication study in an independent cohort would be necessary to confirm our preliminary results.
\end{abstract}

ARTICLE HISTORY

Received 31 January 2018

Revised 9 April 2018

Accepted 20 April 2018

\section{KEYWORDS}

ß2-microglobulin; Alzheimer disease; mild cognitive impairment; serum biomarkers

\section{Introduction}

Imaging, cerebrospinal fluid (CSF) and blood-based biomarkers have the potential to improve the accuracy by which specific causes of dementia can be diagnosed in vivo, provide insights into the underlying pathophysiology, and may be used as inclusion criteria and outcome measures for clinical trials. While a number of imaging and CSF biomarkers are currently used for each of these purposes, this is an evolving field, with numerous potential biomarkers in varying stages of research and development. $\beta 2$-microglobulin $(\beta 2 \mathrm{~m})$ is a low molecular weight $(11.8 \mathrm{KDa})$ non-glycosylated polypeptide. The gene is located on chromosome 15q21.1 and the encoded, functional protein forms the invariant or light $\beta$-chain of HLA class I molecules on the surface of all nucleated cells, in non-covalent association with the $43 \mathrm{KDa}$ heavy $\alpha$-chain of MHC class I antigens (Cunningham and Berggard 1974, Mátrai et al. 2009). The small size of the molecule allows $\beta 2 \mathrm{~m}$ to pass through the glomerular membrane, but normally less of $1 \%$ of protein is excreted in the urine; the remainder is reabsorbed and catabolized in the proximal tubules of the kidney. Nonetheless, it was firstly identified in the urine of patients with renal tubular disease. Since it is processed by glomerular filtration and subsequent tubular reabsorption, increases in serum concentrations are a sensitive marker of impaired renal function. Increased plasma concentrations have also been found in solid tumours, haematological malignancies, autoimmune diseases and infections including AIDS. In particular, $\beta 2 \mathrm{~m}$ is regarded as a robust marker of disease activity and prognosis in lymphoproliferative conditions like myeloma, chronic lymphocytic leukaemia, other lymphomas and infections. Interestingly, some variants of human $\beta 2 \mathrm{~m}$ were associated to increased $\beta 2 \mathrm{~m}$-amyloid deposition and to dialysis-related amyloidosis (Corazza et al. 2004, Corlin et al. 2005, Murray 2008, Foster et al. 2013, Kim et al. 2017). Increased systemic levels of soluble $\beta 2 \mathrm{~m}$ have been implicated in cognitive impairments associated with chronic haemodialysis (Murray 2008, Kim et al. 2017). Despite its important role in prognosis assessment and disease monitoring, relatively few studies are available on its expression in healthy individuals. These reports show remarkable variations in the methods used, age and number of reference individuals and statistical analyses of the data. Further complexity is introduced by the dependence of $\beta 2 \mathrm{~m}$ expression, with respect to race and ethnicity (Cunningham and Berggard 1974, Mátrai et al. 2009). Recently a study investigated the 
association between $\beta 2 \mathrm{~m}$ and incident frailty, concluding with the observation that higher $\beta 2 \mathrm{~m}$ levels were independently associated with greater frailty at baseline in older adults but only slightly associated with greater risk of incident frailty over four years of follow-up (Kim et al. 2017). Furthermore, high $\beta 2 \mathrm{~m}$ levels were recently reported to be associated with cardiovascular disease and mortality in the general population (Astor et al. 2012, Juraschek et al. 2012, Kim et al. 2017, Załęska-Kocięcka et al. 2017). In a study performed on seventy-one patients undergoing aortic valve replacement, patients with $\beta 2 \mathrm{~m}$ increase over the median change $(>0.42 \mathrm{mg} / \mathrm{L})$ experienced a significant in-hospital drop in MMSE $(p=0.005)$. Patients with $\beta 2 \mathrm{~m}$ increase over the median change also failed to improve a delayed-wordrecall domain of the test $(p=0.826)$ while patients with a lower increase improved results in the domain $(p=0.004)$. After six months, MMSE improvement was associated with a significant decrease in $\beta 2 \mathrm{~m}(p=0.042)$ and these authors suggested, the first time in humans, a possible relation between changes in cognition and $\beta 2 \mathrm{~m}$ serum levels (Juraschek et al. 2012, Załęska-Kocięcka et al. 2017). Increased systemic levels of soluble $\beta 2 \mathrm{~m}$ have been implicated in cognitive impairments associated with chronic haemodialysis (Murray, 2008). Moreover, increased soluble $\beta 2 \mathrm{~m}$ has also been detected in the cerebral spinal fluid (CSF) of patients with HIV-associated dementia and Alzheimer's disease (AD) (McArthur et al. 1992, Brew et al. 1996). As reported by Villeda et al., in the brain, $\beta 2 \mathrm{~m}$ and MHC I molecules can act independent of their canonical immune function to regulate normal brain development, synaptic plasticity and behaviour (Smith et al., 2015). Significant changes in the concentration of $\beta 2 \mathrm{~m}$ in mouse plasma have been documented during normal aging, and in the experimental aging model of heterochronic parabiosis. Notably, human genome-wide association studies (GWASs) have linked the MHC locus on chromosome $6 \mathrm{p} 21$ with age-related degenerative diseases, further suggesting an active role for these molecules in age-dependent impairments (Huh et al. 2000, Boulanger and Shatz 2004, Goddard et al. 2007, Shatz 2009, Glynn et al. 2011, Lee et al. 2014, Smith et al. 2015). From the above-mentioned studies, $\beta 2 \mathrm{~m}$ is associated with aging, frailty, some types of cognitive impairment and, limited to CSF, with AD. The experimental studies have confirmed the association with cognitive impairment. Our aim in this study is to provide data about a possible association between cognitive status and $\beta 2 \mathrm{~m}$ plasma levels.

\section{Clinical significance}

- Plasma $\beta 2 \mathrm{~m}$ concentration may improve the accuracy in the identification of individuals with $A D$.

\section{Materials and method}

\section{Populations and plasma samples}

In this retrospective study, we measured the concentration of $\beta 2 \mathrm{~m}$ in a cohort of 245 individuals and compared sex matched, cognitive healthy individuals. Plasma samples were randomly selected form biobanks collected by different research units and programs: healthy controls $(\mathrm{HC})$ samples derived from the longitudinal InveCe.Ab study (Guaita et al. 2013), while the mild cognitive impairment (MCl) and Alzheimer disease (AD) samples were collected from patients population of Fondazione IRCCS $\mathrm{Ca}^{\prime}$ Granda-Ospedale Maggiore Policlinico Milano.

Informed consent was obtained from human subjects according to the institutional review board guidelines at the respective centres, and the study was approved by the Institutional local Ethics Committee.

\section{$A D$ and $\mathrm{MCl}$ patients}

All $\mathrm{AD}$ and $\mathrm{MCl}$ patients underwent a standard battery of examinations, including medical history, physical and neurological examination, screening laboratory evaluation with full blood count, measurement of aspartate and alanine aminotransferase (AST, ALT),VDRL and virology (HIV, hepatitis B and $C$ tests). We genotyped for ApoE\&4 polymorphisms, for the converging role of $A p o E \varepsilon 4$ and $\beta 2 \mathrm{~m}$ for the amyloid beta deposition, a key point for cognitive functions and for $A D$ (Stoppini and Bellotti 2015, Di Battista et al. 2016). Patients with monoclonal gammapathies (MGUS) or active malignant disease and low life expectancy were excluded from the study. A complete neurocognitive and memory evaluation: clinical dementia rating (CDR), mini mental state examination (MMSE), the frontal assessment battery (FAB), the Wisconsin card sorting test (WCST), and the Tower of London test assessed cognitive dysfunctions; imaging was also performed. The presence of significant vascular brain damage was excluded (Hachinski Ischemic Score <4). Lumbar puncture was performed after one-night fasting.

Diagnosis of clinical $\mathrm{MCl}$ was done according to Petersen et al. (2001). These subjects then underwent lumbar puncture and were classified as non-AD MCl, i.e. subjects with CSF amyloid, tau and phospho-tau (P-tau) in the normality range, or prodromal $\mathrm{AD}$ ( $\mathrm{MCl}$ with positive biomarkers, i.e. Amyloid beta $<600 \mathrm{pg} / \mathrm{ml}$, total Tau $>450 \mathrm{pg} / \mathrm{m}$ and P-tau $>61 \mathrm{pg} / \mathrm{ml}$ ) according to current research criteria (Dubois et al. 2007, 2014), AD patients diagnosis fulfilled all criteria (McKhann et al. 1984, 2011), including CSF biomarkers. Nonetheless, we acknowledge that according to the new DSM5 criteria (American Psychiatric Association 2013) we cannot exclude that some of them may develop other dementia than AD.

\section{Controls}

The control group consisted of non-demented volunteers matched for ethnic background, cognitively preserved, without memory and psycho-behavioural dysfunctions (mean MMSE $\geq 28.59$ ). They underwent a neuropsychological cognitive battery, medical examination, standard screening laboratory tests, genetic typing for ApoE\&4 presence/absence. They remained intact and cognitively stable over a minimum follow-up of three years. The first hundred with better scores in the neuropsychological evaluation at the second follow-up were included in the study. 


\section{CSF processing and biomarker determination}

Cerebral spinal fluid samples were obtained in polypropylene tubes by lumbar puncture at the L4/L5 or L3/L4 interspace, centrifuged at $4{ }^{\circ} \mathrm{C}$ at $2000 \mathrm{~g}$. The serum and CSF samples were removed and dispensed in aliquots of $400 \mu \mathrm{L}$ into cryotubes. Specimens were stored at $-80^{\circ} \mathrm{C}$ until use. $A \beta 42$, tau and P-tau CSF levels were determined with human specific ELISA kits (Innogenetics, Ghent, Belgium), as previously reported (Andreasen and Blennow 2005).

\section{B2 Microglobulin analysis}

$\beta 2 \mathrm{~m}$ was measured from frozen plasma samples (EDTA, $-20^{\circ} \mathrm{C}$ ) (Lee et al. 2014) collected at the baseline study visit, with the use of a solid-phase, two site chemiluminescent immunometric assay on Immulite 2000 Automatic analyzer (Siemens Healthcare Diagnostics $\mathrm{GmbH}$, Eschborn, Germany).

\section{Statistical analysis}

We used one-way ANOVA for the continuous variables. In case of a significant $p$-value $(<0.05)$, we performed a post hoc comparison (Bonferroni). For categorical variable we used the Chi square or Fisher Exact test when appropriate. The independent role of socio-demographic characteristics on outcomes was also verified, by multinomial regression analysis. Due to the different mean age of the three groups, age was introduced in the regression as main covariate: alone (first model), associated with gender (second model), associated with gender and ApoEs4 (third model), for undoing the effect of age on the possible association with the three diagnostic groups of the outcome ( $\beta 2 \mathrm{~m}$ level). In all cases a $p$-value less than 0.05 was considered significant. Data were processed using SPSS $11+$ release.

\section{Patients and controls flow charts}



\section{Control subjects (InveCe.Ab study): flow chart}

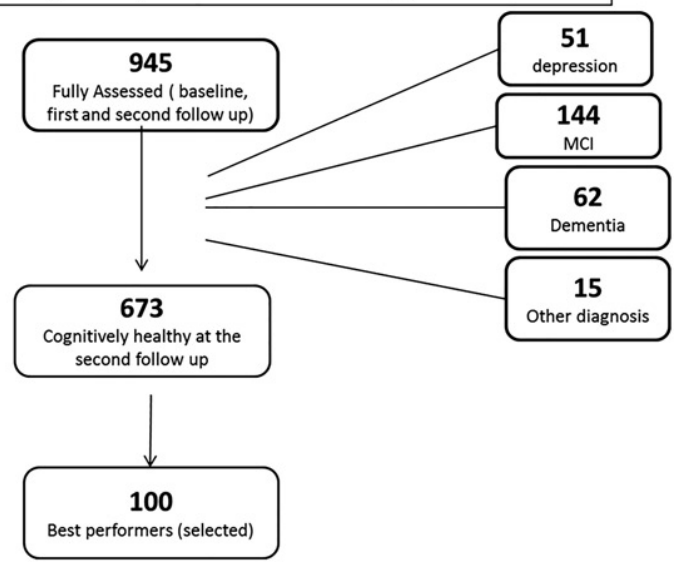

\section{Results}

Serum levels of $\beta 2 \mathrm{~m}$ were measured in $100 \mathrm{HC}, 45 \mathrm{MCl}$ and 100 AD. Demographic and clinical characteristics of the three groups are reported in Table 1.

Mild cognitive impairment patients were significantly younger than $H C$ and $A D$, while there was no difference between the last two. Gender distribution was similar among the groups. APOE $\varepsilon 4$ was present at higher percentage in the $M C l$ and $A D$ groups, but with a borderline significance $(p=0.078)$.

No interaction between gender and $\mathrm{APOE} \varepsilon 4$ was detected. There was no effect of age on gender or APOE 44 distribution, but a significant linear correlation between age and $\beta 2 \mathrm{~m}$ serum levels ( $r$ " $=0.247 ; p<0.001$ ). The difference in $\beta 2 \mathrm{~m}$ mean levels among $\mathrm{HC}, \mathrm{MCl}$ and $\mathrm{AD}$, are reported in Figure 1 and in Table 2 , with post-hoc analysis. AD patients showed values higher than $\mathrm{MCl}$ and $\mathrm{HC}(p<0.001$ and $p=0.033$, respectively), while there was no significant difference between $\mathrm{HC}$ and $\mathrm{MCl}$.

Due to the importance of age in the serum levels of $\beta 2 \mathrm{~m}$, we performed a multinomial regression with $A D$ as reference with respect to $\mathrm{HC}$ and $\mathrm{MCl}$ and $\beta 2 \mathrm{~m}$ plasma levels as dependent variable, then applying three models with age, age + gender, age + gender $+\operatorname{APOE} \varepsilon 4$. In all models $\beta 2 \mathrm{~m}$ levels are significantly higher in $A D$ patients compared to $\mathrm{MCl}$ subjects (firstst model: $p<0.001$; OR: 0.886 ; $95 \% \mathrm{Cl}$ : $0.833-0.943$; second model: $p=0.005$; OR: 0.999 ; $95 \% \mathrm{Cl}: 0.998-1.000$; third model: $p=0.019$; OR: 0.999; 95\%Cl: 0.998-1.000). Compared to HC, AD patients show higher levels of $\beta 2 \mathrm{~m}$ levels, significantly for first two models and borderline for the third (first model: $p=0.039$; OR: 0.999; 95\%Cl: 0.999-1.000; second model: $p=0.04$; OR: 0.999; 95\%Cl: 0.999-1.000; third model: $p=0.055$; OR: 1.000; 95\%Cl: 0.999-1.000).

Table 1. Demographic characteristics and presence of ApoE\&4 in the three groups.

\begin{tabular}{lccc}
\hline & $\mathrm{HC}(\mathrm{N}: 100)$ & $\mathrm{MCl}(\mathrm{N}: 45)$ & $\mathrm{AD}(\mathrm{N}: 100)$ \\
\hline Years of age & 72.02 (SD: 1.271$)$ & 67.34 (SD: 8.402) & 73.17 (SD: 7.411) \\
Female (\%) & 52 & 53.3 & 61 \\
ApoE\&4 (\%) & 19 & 34.2 & 31 \\
\hline
\end{tabular}

The mean age are lower for $\mathrm{MCl}(p<0.001)$; the gender and ApoE4 percentages are not different in the three groups (HC: Healthy Controls; MCl: Mild Cognitive Impairment; AD: Alzheimer's Disease). 


\section{Discussion}

Alzheimer's disease can only be definitively diagnosed at autopsy since its manifestations of senile plaques and neurofibrillary tangles throughout the brain cannot yet be fully captured with current imaging technologies. The identification and validation of biomarkers for diagnosing $A D$ and other forms of dementia are increasingly important. To date, ELISA measurement of $\beta$-amyloid peptide (1-42), total tau and phospho-tau-181 in cerebrospinal fluid (CSF) is the most advanced and accepted method to diagnose probable $A D$ with high specificity and sensitivity.

Besides identifying markers that distinguish $A D$ from controls, there has been a recent drive to identify better biomarkers that can predict the rates of cognitive decline and neocortical amyloid burden in those who exhibit preclinical, prodromal, or clinical AD. In addition to studying CSF biomarkers, researchers have sought less invasive sources, such as blood (O'Bryanta et al. 2017).

Although saliva or urine can be easily collected, blood analysis is the gold standard (Blennow 2017); yet, it is still unknown how the concentration of analytes in the blood directly correlates with pathological changes in the brain, especially in AD. The search for blood biomarkers that correlate with $A D$ should therefore begin with accepted CSF markers, such as $A \beta$ and tau-related biomarkers, and further include factors involved in inflammation, protein aging and cell death, and cerebrovascular dysfunctions. It seems very plausible that, at some point in the next years, the combination of different blood-derived $A D$ biomarkers leads to the definition of a patient-specific signature. The pilot European trial on $A D$ neuroimaging Initiatives (E-ADNI) measured both CSF and plasma derived $A \beta$ and found that higher diagnostic accuracy was obtained with frozen than fresh samples. Obtaining blood samples is relatively painless and inexpensive, giving potential blood-based biomarkers further advantage over the CSF-based markers. Furthermore some of the blood biomarkers appear to be just as diagnostically accurate as the CSF-based and genetic biomarkers though further validation is warranted. Useful biomarkers could predict the progression of $\mathrm{MCl}$ to $\mathrm{AD}$ so that early preventative treatment can be delivered to AD-presymptomatic patients.

Acknowledging that peripheral biomarkers (blood or otherwise) of brain disorders are more difficult to identify and lockdown, there are many potential advantages for blood-based $A D$ biomarkers, including, but not limited to, primary care screening, diagnostics, predictive risk (i.e. risk for incident $A D$, risk for progression from $M C l$ to $A D$ ), disease monitoring, stratification into clinical trials, and pharmacodynamics or treatment response monitoring (positive or adverse). Indeed, an important potential of AD blood biomarkers could be to increase the likelihood of subjects being positive on more expensive (e.g. positron emission tomography [PET] imaging) or invasive (lumbar puncture for CSF sampling) biomarkers used later to determine trial eligibility (McKhann et al. 1984, 2011, Andreasen and Blennow 2005).

In this work, we provide a preliminary evidence of different plasma concentrations of $\beta 2 \mathrm{~m}$ in patients with $A D$ respect to $\mathrm{MCl}$ and healthy controls. Though old age is a main factor for serum levels of $\beta 2 \mathrm{~m}$, we found that the difference remained significant after controlling for age. Nevertheless, the effect is weak, with OR 0.99 that cannot allow to draw other than

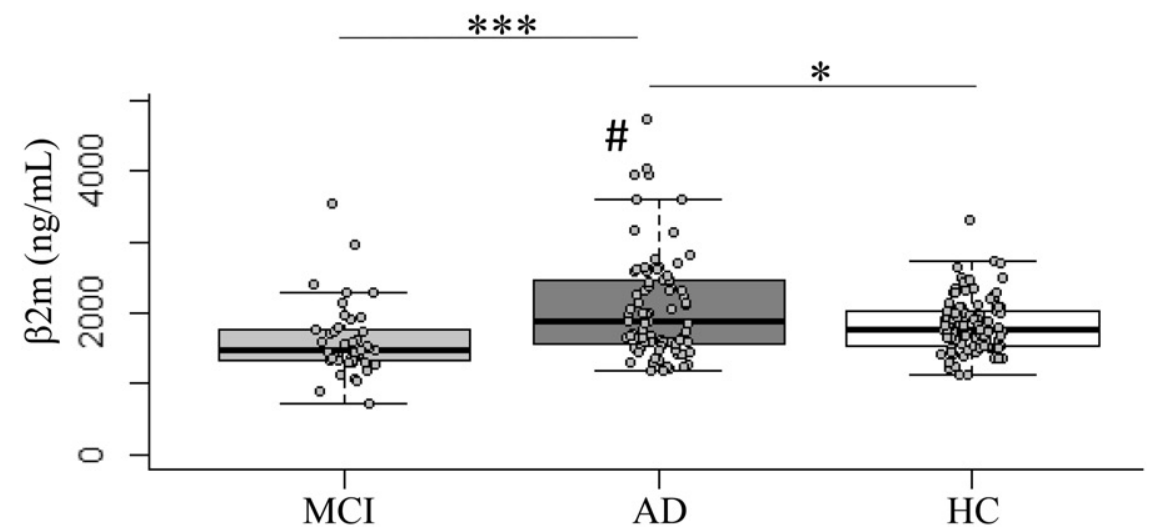

Figure 1. Box and whiskers plot of $\beta 2 \mathrm{~m}$ serum levels in $M C l, A D$, and $H C$. Mean concentration of $\beta 2 \mathrm{~m}$ in $\mathrm{MCl} 1613 \mathrm{ng} / \mathrm{mL} \pm 509 \mathrm{SD}, A D 2063 \mathrm{ng} / \mathrm{mL} \pm 852 \mathrm{SD}$, and HC $1832 \mathrm{ng} / \mathrm{mL} \pm 384$ SD. ${ }^{*} p=0.033 ;{ }^{* * *} p<0.001$, ANOVA post hoc analyses (Bonferroni). \#An outlier value of $6955 \mathrm{ng} / \mathrm{mL}$ in the AD population has been omitted from the graph.

Table 2. $\beta 2 \mathrm{~m}$ mean difference between the three groups.

\begin{tabular}{|c|c|c|c|c|c|}
\hline \multicolumn{6}{|c|}{ Dependent variable: $\beta 2 \mathrm{~m}$ ( $\mathrm{ng} / \mathrm{mL})$} \\
\hline & \multirow[b]{2}{*}{$\beta 2 \mathrm{~m}(\mathrm{ng} / \mathrm{mL})$ Mean difference } & \multirow[b]{2}{*}{ Std. Error } & \multirow[b]{2}{*}{ Sig. } & \multicolumn{2}{|c|}{ 95\% Confidence interval } \\
\hline & & & & Lower bound & Upper bound \\
\hline $\mathrm{HC}-\mathrm{MCl}$ & 219.88 & 114.13457 & 0.166 & -55.2674 & 495.0296 \\
\hline$H C-A D$ & $-230.41^{*}$ & 89.91962 & 0.033 & -447.1826 & -13.6374 \\
\hline$M C l-A D$ & $-450.29 * * *$ & 114.13457 & $<0.001$ & -725.4396 & -175.1426 \\
\hline
\end{tabular}

In ANOVA post hoc analyses (Bonferroni) $A D$ subjects show higher mean levels of $\beta 2 \mathrm{~m}$ than $\mathrm{MCl}$ and $\mathrm{HC}$ $\left({ }^{* * *} p<0.001\right.$ and ${ }^{*} p=0.033$, respectively). MCI do not show difference from HC $(p=0.166)$. 
preliminary considerations. The slightly reduction of statistically significant $A D-H C$ difference after introducing APOE $\varepsilon 4$ in regression model is explained by the limited number of the sample and by the higher $\beta 2 \mathrm{~m}$ level, though non-significant, in the APOE $\varepsilon 4$ + subjects.

Actually our study has some important limitations. First of all the number of participants is very small in the $\mathrm{MCl}$ group, that was and second, age that is significantly different for $\mathrm{MCl}$ compared to $A D$ and healthy controls. Nevertheless, strengths include the diagnosis made with the use of biomarkers and the follow up of controls, that remained cognitively intact over a minimum follow up of three years. Aging remains the most dominant risk factor for dementia-related neurodegenerative diseases, such as AD. The experimental evidences of the Villeda paper showed that $\beta 2 \mathrm{~m}$ could affect hippocampal functions in mice, in fact this protein, as a circulating factor negatively regulates cognitive and regenerative function in the adult hippocampus in an age-dependent manner. $\beta 2 \mathrm{~m}$ is elevated in the blood of aging humans and mice, and is increased within the hippocampus of aged mice and in the model of young heterochronic parabionts. These author with their data indicate that systemic $\beta 2 \mathrm{~m}$ accumulating in aging blood promotes age-related cognitive dysfunction and impaired neurogenesis, in part via MHC I, suggesting $\beta 2 \mathrm{~m}$ may be targeted in old age (Smith et al. 2015).

Herein, we showed that plasma $\beta 2 \mathrm{~m}$ levels are specifically increased in $A D$, independent of the phase (prodromal or fully symptomatic $A D$ ). Notably, clinical diagnosis of patients included in the study was supported by the analysis of CSF biomarkers, which allowed us to classify patients, basing on the demonstration of an amyloid and tau underlying pathology, in $\mathrm{MCl}$ due to $\mathrm{AD}$ (prodromal $\mathrm{AD}$ ) from $\mathrm{MCl}$ due to other causes. The use of such biomarkers allows to discriminate between $\mathrm{MCl}$ due to $A D$, i.e. prodromal $A D$, from $\mathrm{MCl}$ due to other causes (non-AD MCl) with a very high accuracy (Blennow 2017, O'Bryanta et al. 2017), and represents the best surrogate biomarker to be used in the absence of the demonstration of the pathology at autopsy. In order to explain the discrepancy between $\mathrm{MCl}$ and $\mathrm{AD}$, we speculate that, in according with the new criteria of classification (McKhann et al. 1984, 2011, Andreasen and Blennow 2005, Dubois et al. 2007, 2014, American Psychiatric Association 2013), a clinically defined MCI with the demonstration of CSF biomarkers (low $A \beta$ and high tau) is to consider as 'prodromal' $A D$, while if the biomarker do not fit with $A D$, $\mathrm{MCl}$ is not to correlate or attribute to $A D$ pathology.

Recent experimental evidences have suggested the pathogenetic contribution of glia to progression of disease; in particular when it has lost the neuronal homeostatic functions and acquired the pro-inflammatory phenotype. Activated microglial cells express MHC class I and $\beta 2 \mathrm{~m}$ on their surface, and in response to the pathogenic noxae can amplify the inflammatory cascade responsible of exacerbation of neurodegenerative process (Boche and Nicoll 2008, McQuillan et al. 2010).

\section{Conclusions}

We measured plasma levels of $\beta 2 \mathrm{~m}$ in $100 \mathrm{HC}, 100 \mathrm{AD}$ and $45 \mathrm{MCl}$. The study revealed a statistically significant difference of $\beta 2 \mathrm{~m}$ concentration in patients with advanced $A D$ respect to $\mathrm{MCl}$ and a borderline difference with $\mathrm{HC}$. This suggests that plasma $\beta 2 \mathrm{~m}$ may be a useful biomarker to distinguish subjects with $A D$ from $\mathrm{MCl}$ and possibly $\mathrm{HC}$. However, a replication study in a larger and independent cohort would be necessary to confirm our preliminary results.

\section{Disclosure statement}

No potential conflict of interest was reported by the authors.

\section{Acknowledgements}

We are grateful to Daniela Busi and Michela Faustini for technical assistance with the determination of $\beta 2 \mathrm{~m}$ levels.

\section{ORCID}

Dario Finazzi iD http://orcid.org/0000-0001-5176-2839

Emanuela Oldoni (iD http://orcid.org/0000-0002-9105-2601

Elio Scarpini (ID http://orcid.org/0000-0002-6395-2119

Daniela Galimberti ID http://orcid.org/0000-0002-9284-5953

Antonio Guaita (D) http://orcid.org/0000-0003-3954-5932

\section{References}

American Psychiatric Association. 2013. Diagnostic and statistical manual of mental disorders (DSM-5), 5th ed. Washington, DC: American Psychiatric Association.

Andreasen, N. and Blennow, K., 2005. CSF biomarkers for mild cognitive impairment and early Alzheimer's disease. Clinical neurology and neurosurgery, 107, 165-173.

Astor, B.C., et al., 2012. Novel markers of kidney function as predictors of ESRD, cardiovascular disease, and mortality in the general population. American journal of kidney diseases, 59, 653-662.

Blennow, K., 2017. A Review of fluid Biomarkers for Alzheimer's Disease: Moving from CSF to Blood. Neurology and therapy, 6, 15-24.

Boche, D. and Nicoll, J.A., 2008. The role of the immune system in clearance of $A \beta$ from the brain. Brain pathology, 18, 267-278.

Boulanger, L.M. and Shatz, C.J., 2004. Immune signalling in neural development, synaptic plasticity and disease. Nature reviews neuroscience, 5 , 521-531.

Brew, B.J., et al., 1996. Predictive markers of AIDS dementia complex: CD4 cell count and cerebrospinal fluid concentrations of $\beta 2$ microglobulin and neopterin. Journal of infectious diseases, 174, 294-298.

Carrette, O., et al., 2003. A panel of cerebrospinal fluid potential biomarkers for the diagnosis of Alzheimer's disease. Proteomics, 3, 1486-1494.

Corazza, A., et al., 2004. Properties of some variants of human 32 -microglobulin and amyloidogenesis. Journal of biological chemistry, 279, 9176-9189.

Corlin, D.B., et al., 2005. Quantification of cleaved b2-microglobulin in serum from patients undergoing chronic hemodialysis. Clinical chemistry, 51, 1177-1184.

Cunningham, B.A. and Berggard, I., 1974. Structure, evolution and significance of beta-2-microglobulin. Immunological reviews, 21, 3-14.

Di Battista, A.M., Heinsinger, N.M., and Rebeck, G.W., 2016. Alzheimer's disease genetic risk factor APOE- $\varepsilon 4$ also affects normal brain function Current Alzheimer research, 13 (11), 1200-1207.

Dubois, B., et al., 2007. Research criteria for the diagnosis of Alzheimer's disease: revising the NINCDS-ADRDA criteria. Lancet neurol, 6 (8), 734-746.

Dubois, B., et al., 2014. Advancing research diagnostic criteria for Alzheimer's disease: the IWG-2 criteria. Lancet neurol, 13 (6), 614-629. 
Foster, M.C. et al., 2013. Novel filtration markers as predictors of all-cause and cardiovascular mortality in US adults. American journal of kidney diseases, 62 (1), 42-51.

Glynn, M.W., et al., 2011. MHC Class I negatively regulates synapse density during the establishment of cortical connections. Nature neuroscience, 14 (4), 442-451.

Goddard, C.A., Butts, D.A., and Shatz, C.J., 2007. Regulation of CNS synapses by neuronal MHC class I. Proc. Proceedings of the national academy of sciences of the United States of America, 104, 6828-6833.

Guaita, A., et al., 2013. Brain aging and dementia during the transition from late adulthood to old age: design and methodology of the "Invece.Ab" population-based study. BMC geriatrics, 13 (1), 98.

Huh, G.S., et al., 2000. Functional requirement for class I MHC in CNS development and plasticity. Science (New York, N.Y.), 290, 2155-2159.

Juraschek, S.P., et al., 2012. The effects of freeze-thaw on $\beta$-trace protein and $\beta 2$ - microglobulin assays after long-term sample storage. Clinical biochemistry, 45 (9), 694-696.

Kim, M., et al., 2017. Association between serum $\beta 2$-microglobulin levels and prevalent and incident physical frailty in community-dwelling older women. JAGS, 65 (4), e83-e88.


by MHC class I H2-Db. Nature, 509, 195-200.

Mátrai, Z., et al., 2009. Serum $\beta 2$-microglobulin measured by immunonephelometry: expression patterns and reference intervals in healthy adults. Clinical chemistry and laboratory medicine, 47, 585-589.

McArthur, J.C., et al., 1992. The diagnostic utility of elevation in cerebrospinal fluid beta 2-microglobulin in HIV-1 dementia. Multicenter AIDS Cohort Study. Neurology, 42, 1707-1712.

McKhann, G., et al., 1984. Clinical diagnosis of Alzheimer's disease: report of the NINCDS-ADRDA Work Group under the auspices of Department of Health and Human Services Task Force on Alzheimer's disease. Neurology, 34 (7), 939-944.

McKhann, G.M., et al., 2011. The diagnosis of dementia due to Alzheimer's disease: recommendations from the National Institute on Aging-Alzheimer's Association workgroups on diagnostic guidelines for Alzheimer's disease. Alzheimers dement, 7 (3), 263-269.

McQuillan, K., Lynch, M.A., and Mills, K.H.G., 2010. Activation of mixed glia by $A \beta$-specific Th1 and Th17 cells and its regulation by Th2 cells. Brain, behavior, and immunity, 24, 598-607.

Murray, A.M., 2008. Cognitive impairment in the aging dialysis and chronic kidney disease populations: an occult burden. Advances in chronic kidney disease, 15, 123-132.

O'Bryanta, S.E., et al., 2017. Blood-based biomarkers in Alzheimer disease: current state of the science and a novel collaborative paradigm for advancing from discovery to clinic. Alzheimer's \& dementia, 13, 45-58.

Petersen, R.C., et al., 2001. Practice parameter: early detection of dementia: mild cognitive impairment (an evidence-based review). Report of the Quality Standards Subcommittee of the American Academy of Neurology. Neurology, 56 (9), 1133-1142.

Shatz, C.J., 2009. MHC class I: an unexpected role in neuronal plasticity. Neuron, 64, 40-45.

Smith, L.K., et al., 2015. $\beta 2$-microglobulin is a systemic pro-aging factor that impairs cognitive function and neurogenesis. Nature medicine, 21 (8), 932-937.

Stoppini, M. and Bellotti, V., 2015. Systemic amyloidosis: lessons from $\beta 2$-microglobulin. Journal of biological chemistry, 290 (16), 9951-9958.

Załeska-Kociecka, M., et al., 2017. Role of $\beta 2$-microglobulin in postoperative cognitive decline. Biomarkers in medicine, 11 (3), 245-253. 